\title{
Note de correction : Comment les soignants-éducateurs ont-ils adapté leurs pratiques de I'ETP durant la crise du COVID-19? Une enquête descriptive sur 714 programmes d'ETP
}

\section{Educ Ther Patient/Ther Patient Educ 2020, 12, 20207, https://doi.org/10.1051/tpe/2021001}

Pascale Lafitte ${ }^{1,5, *}$, Benoît Pétré ${ }^{2,5}$, Xavier de la Tribonnière ${ }^{3,4,5}$ et Rémi Gagnayre en $^{4,5}$

${ }^{1}$ UTEP 65, Hôpital le Montaigu, 65200 Astugue, France

2 Département des Sciences de la Santé publique, Université de Liège, Liège, Belgique

3 Unité transversale d'éducation du patient (UTEP), CHU de Montpellier, Université de Montpellier, 34000 Montpellier, France

${ }^{4}$ Laboratoire Educations et Pratiques de Santé UR 3412, Université Sorbonne Paris Nord, 93000 Bobigny, France

5 Société d'Education Thérapeutique Européenne (SETE**), 47000 Agen, France

Demande de correction soumise et acceptée le 15 mars 2021

Des erreurs ont été introduites dans la version originale de l'article publié dans Educ Ther Patient/Ther Patient Educ 2020, 12, 20207. Suite à des erreurs dans le tableau III, l'article a été entièrement republié. L'éditeur s'excuse pour la gêne occasionnée.

\footnotetext{
*Auteur de correspondance : plafitte@hopital-le-montaigu.com

${ }^{* *}$ https://www.socsete.org.
} 